\title{
DRONE DATA ATMOSPHERIC CORRECTION CONCEPT FOR MULTI- AND HYPERSPECTRAL IMAGERY - THE DROACOR MODEL
}

\author{
Daniel Schläpfer ${ }^{1 *}$, Christoph Popp ${ }^{1}$, Rudolf Richter ${ }^{2}$ \\ ${ }^{1}$ ReSe Applications LLC, Langeggweg 3, CH-9500 Wil SG, Switzerland; daniel@ rese.ch, popp@ rese.ch \\ ${ }^{2}$ German Aerospace Center (DLR), Earth Observation Center, D-82234 Wessling, Germany; rudolf.richter@ dlr.de
}

Commission III, WG III/4

KEY WORDS: Atmospheric Correction, Reflectance Retrieval, Drone data processing, irradiance correction

\begin{abstract}
:
Remote sensing with unmanned aerial vehicles (UAVs) is a fast and cost-efficient tool for mapping and environmental monitoring. The sensors are operated at low flight altitudes, usually below $500 \mathrm{~m}$ above ground, leading to spatial resolutions up to the centimeter range. This type of data causes new challenges in atmospheric compensation and surface reflectance retrieval. Based on these specific boundary conditions, a new drone based atmospheric correction concept (DROACOR) is proposed, which is designed for currently available UAV based sensors. It is suited for multispectral visible/near infrared sensors as well as hyperspectral instruments covering the $400-1000 \mathrm{~nm}$ spectral region or the $400-2500 \mathrm{~nm}$ spectrum. The goal of the development is a fully automatic processor which dynamically adjusts to the given instrument and the atmospheric conditions. Optionally, irradiance measurements from simultaneously measured cosine receptors or from in-field reference panels can be taken into account to improve the processing quality by adjusting the irradiance parameter or by performing an in-flight vicarious calibration. Examples of DROACOR processing results are presented for a multispectral image data set and a hyperspectral data set, both acquired at variable flight altitudes. The resulting spectra show the applicability of the methods for both sensor types and an accuracy level below $2.5 \%$ reflectance units.
\end{abstract}

\section{INTRODUCTION}

During the last few years, drone-borne sensors have been widely used in geology, exploration, and agriculture. A typical example of multispectral UAV sensors is the 5-channel Micasense Red Edge sensor (blue, green, red, red-edge, NIR) (Micasense Inc., 2019), whereas examples of hyperspectral instruments are Headwall Nano (Headwall Photonics Inc., 2019) (272 channels from $400 \mathrm{~nm}$ to $1000 \mathrm{~nm}$ ), Hyspex Mjolnir (Norsk Elektro Optikk AS, 2019) (488 channels from $400 \mathrm{~nm}$ to $2500 \mathrm{~nm}$ ), and the AISA AFX-10 sensor (Specim Spectral Imaging Ltd., 2020) ( 220 channels, $400 \mathrm{~nm}-1000 \mathrm{~nm}$ ). Using such systems, image acquisition is possible for clear and cloudy conditions and in areas difficult to access. While airborne data acquisitions usually cover altitudes from $500 \mathrm{~m}$ to $5 \mathrm{~km}$ above ground, and up to $20 \mathrm{~km}$ in extreme cases, UAV platforms typically operate from $10 \mathrm{~m}$ to $500 \mathrm{~m}$ above ground. The low UAV altitude and the related very high spatial resolution poses new requirements on the surface reflectance retrieval. Reflectance retrieval methods for such image data is often based on empirical normalisation to in-situ reference panel reflectances (Aasen, Bolten, 2018). Radiometric block adjustment and multi-image calibration are possible advanced solutions to this problem (Eija Honkavaara, Ehsan Khoramshahi, 2018, Guo et al., 2019). Such statistical methods lead to smooth and well balanced mosaics but bear the inherent risk of drifts in the absolute surface reflectance information due to image-to-image error propagation.

Physical methods and radiative transfer calculations have to account for the short optical path of observation. Immediate consequences concern the small water vapor column below the sensor platform, whereas the largest amount (i.e., above the

\footnotetext{
* Corresponding author contact: daniel@ rese.ch
}

platform) still has to be considered for modelling the irradiance term. The same applies to the aerosol load: the aerosol scattering of the path ground-to-UAV is small, but scattering/extinction properties of the higher aerosol layers influence both the diffuse and the direct solar flux on the ground. This means the directly backscattered path radiance plays a minor role, but the atmospheric influence still exists for the sun-toground path and needs to be corrected. Moreover, the influence of the adjacency effect is reduced at low flight altitudes compared to traditional airborne imagery (Richter et al., 2006). Due to the low spatial extent of the imagery and the irradiancedriven signatures, the aerosol and water vapor effects may be assumed being spatially constant for the processing. Thus, an image-derived average value for column water vapor and aerosol optical thickness can be used for the processing instead of spatial distribution maps.

Other factors that have to be considered are the availability of absolute or relative radiometric calibration and the specific properties of sensors used in drone-based remote sensing (Aasen, Bolten, 2018, Aasen et al., 2018). The latter includes the strong sensor motions and self-adjusting sensor parameters in flight (such as variable integration times). Because of all these differences, the traditional processing chain for airborne optical instruments, which is often based on the ATCOR4 method (Richter, Schläpfer, 2002, Richter, Schläpfer, 2019) or similar approaches, is not adequate. A concept of a 'drone atmospheric correction method' (DROACOR) is proposed hereafter which considers the above-mentioned peculiarities of drone imagery and shall lead to an operational radiative transfer based atmospheric correction tool for drone data. A prototype of the outlined procedure has been implemented and first results are shown for both multispectral and hyperspectral instruments. The major processing steps are outlined below. 


\section{METHOD}

The presented method consists of five major processing steps as depicted in Figure 1. The process shall be applicable for frame imagers as well as for line scanners having at least the standard blue-red-green-near infrared spectral bands. The separate procedures may vary significantly, depending on the sensor type and the availability of auxiliary measurements. Details of the proposed methodological steps are described hereafter.

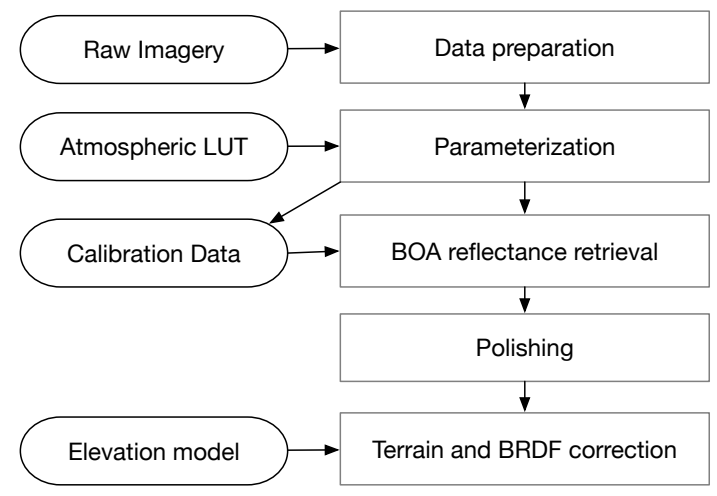

Figure 1. Processing flowchart of the DROACOR method

\subsection{Data Preparation}

The initial data preparation steps include image data import and transformation to co-registered band sequential formats. Furthermore, meta data is read from standard auxiliary data streams. Required meta data are: time, location (Lat/Lon), solar angles, observation angles, sensor internal geometry, terrain height and platform altitude. The data itself has either to be laboratory-calibrated to $\left[\mathrm{mW} /\left(\mathrm{cm}^{2} \mathrm{sr} \mu \mathrm{m}\right)\right]$ or accompanied with panel reflectance information, visible in one image of the campaign series. Essential processing parameters such as height above ground, solar zenith and azimuth angles and calibration information are written to JSON-formatted parameter files which are used for further processing.

\subsection{Parametrization}

The parametrization includes various steps where additional information is retrieved from the image directly. For all instruments, two steps are typically performed:

- estimate of the aerosol optical thickness (AOT) at $550 \mathrm{~nm}$ from signatures in the blue spectral channel, and

- optional radiometric in-flight calibration based on reference panels.

The inflight radiometric calibration is using the panel reflectance information to derive updated sensor calibration information (Cattrall et al., 2002) for both radiometric gain and dark current offset. The calibration coefficients are assumed being constant if applied to a time series of images.

For imaging spectrometers, two more processes are recommended:

- spectral shift detection and correction based on atmospheric absorption features and adaption of corresponding LUTs (Richter et al., 2011), and
- image based estimate of average total column atmospheric water vapor.

Water vapor and aerosol optical thickness (AOT at $550 \mathrm{~nm}$ ) are retrieved as image average value by spectral fitting techniques (Thompson et al., 2018). Using this information, the sensorspecific atmospheric look-up-table (LUT) is created as a subset of the high spectral resolution LUT. The method uses a generic atmospheric LUT based on the LibRadtran (Mayer et al., 2019) radiative transfer code. The parameter space of the LUT is summarized in Table 1. It covers the typical range of UAV data acquisitions and is suited for currently known UAV systems.

\begin{tabular}{|l|c|}
\hline Property & Parameter Range \\
\hline spectral range: & $365-2550 \mathrm{~nm}$ \\
spectral resolution: & $1.0 \mathrm{~nm}$ \\
ground elevations: & $0,500,1000,2000,4000 \mathrm{~m}$ a.s.l. \\
UAV altitudes: & $10,100,200,500 \mathrm{~m}$ above ground \\
solar zenith angles: & $10^{\circ}$ to $70^{\circ}$, increment $10^{\circ}$ \\
visibility range (AOT): & $10-80 \mathrm{~km}$ \\
water vapor columns; & $0.4-5 \mathrm{~cm}$, for sea level \\
\hline
\end{tabular}

Table 1. Specifications of the DROACOR LUT used for radiative transfer based atmospheric compensation.

\subsection{Reflectance Retrieval}

In this main processing step, the bottom of atmosphere (BOA) apparent reflectance is calculated from imagery. The reflectance retrieval is based on absolute radiometric calibration of an instrument from laboratory or from inflight calibration information. This may be enhanced by irradiance sensors if either the sensor calibration is inaccurate (or missing) or if data has been acquired in cloudy conditions. The bottom of atmosphere reflectance retrieval from calibrated at-sensor radiance $L_{s}$ is done by the transformation:

$$
\rho=\frac{\pi\left(d^{2} L_{s}-L_{p}\right)}{\left(E_{d i r}+E_{d i f}\right)\left(\tau_{o f f}+\tau_{d i f}\right)},
$$

where $d$ is the relative earth-sun distance (astronomical units), $L_{p}$ is the (small) path scattered radiance, $E_{d i r}$ is the direct solar ground flux on the ground, $E_{d i f}$ the diffuse flux, $\tau_{o f f}$ is the off-nadir transmittance and $\tau_{\text {dif }}$ the diffuse ground-to-sensor transmittance. The total solar flux may be adapted based on an irradiance sensor within the atmospheric correction equation (Richter, Schläpfer, 2019). Note that the reflectance retrieval as of Eq. (1) does not include the influence of adjacency effects from nearby object by atmospheric scattering, which are assumed being negligible due to the low flight altitudes.

\subsection{Spectral Polishing and Interpolation}

For imaging spectrometers, the retrieved spectra are to be optimized to improve the comparability to reference spectra. Spectral polishing/filtering process are employed for the removal of artefacts (Schläpfer, Richter, 2011). Furthermore, spectral bands within strongly absorbing atmospheric features are interpolated for the reconstruction of a continuous spectrum. The latter is of higher relevance for SWIR imagers where large portions of the spectrum are affected by strong atmospheric absorption. No such methods are required for multispectral imagery. 


\subsection{Terrain and BRDF correction}

The terrain effects can be corrected after BOA reflectance retrieval, as only little cross-talk through the atmosphere is affecting the imagery. The goal of this step is the transformation of apparent bottom of atmosphere reflectance to spectral albedo values (i.e. bihemispherical reflectances). This step can be approximated in many ways, outlined by (Sola et al., 2016). We propose to use a modified Minnaert approach (Richter, Schläpfer, 2019), which avoids overcorrection of BRDF effects on the incident direction. For wide FOV instruments, the BREFCOR method may be applied to correct for BRDF effects depending on the observation angle (Schläpfer et al., 2015).

\section{RESULTS}

Two examples of DROACOR processing are presented, the first one for multispectral imagery (Micasense), the second for hyperspectral data (Hyperspec Nano). Both systems are not laboratory-calibrated. Thus, inflight calibration based on reference panels has to be applied in the course of the atmospheric correction. Please note that the presented results are based on LUTs calculated with the MODTRAN 5.4 (Berk et al., 2005) radiative transfer code, which are to be replaced by LibRadtran-based tables in a later stage.

\subsection{Micasense RedEdge Multispectral Instrument}

The Micasense Rededge-M test data has been taken September 11th 2018 in Wil, Switzerland. It was flown over a sports area while the flight altitude was varied continuously between 0 and $140 \mathrm{~m}$ above ground level. The solar zenith angle varied only slightly between 45.4 and 45.7 degrees within the five minutes of data acquisition. In-field reference standard cardboard panels are used for irradiance calibration and for validation purposes. The panel reflectances are measured in-field using the oceanoptics USB-2000 hand held spectrometer. A sample scene is shown in Figure 2. The data is coregistered and system calibrated using image-based meta data information, including correction for integration time variations and dark signal. Inflight calibration of the sensor was done using one data set at an altitude of $37 \mathrm{~m}$ above ground on the basis of a dark reference panel (about 5\% reflectance) and a bright reference panel (about 50\% reflecting). The respective calibration information is then applied in the processing for all other data sets which results in the reflectance spectra as shown in Figure 3.

The evaluation of this sample data set shows consistent reflectance retrieval results for all flight altitudes between 30 and $140 \mathrm{~m}$ above ground. The mean deviation from the reference panel reflectance is typically between 1.5 and $3 \%$ reflectance units (compare Table 2). This absolute error is increasing with brightness, most likely due to inaccuracies of the radiometric gain from inflight calibration. The variability may be attributed to the sensor motion during data acquisition and the automatic adaption of the integration time of the Micasense instrument. The latter leads to inconsistencies in terms of the dark current level and instrument gain which apparently are not fully accounted for in the current data preprocessing. For low sensor altitudes below $30 \mathrm{~m}$, the retrieved reflectances deviate significantly from higher altitude data acquisitions, specifically in the near infrared band. This effect may be attributed to sensor-specific effects such as variability of integration times and dark signal which may be inaccurate in the applied raw data processing. Another

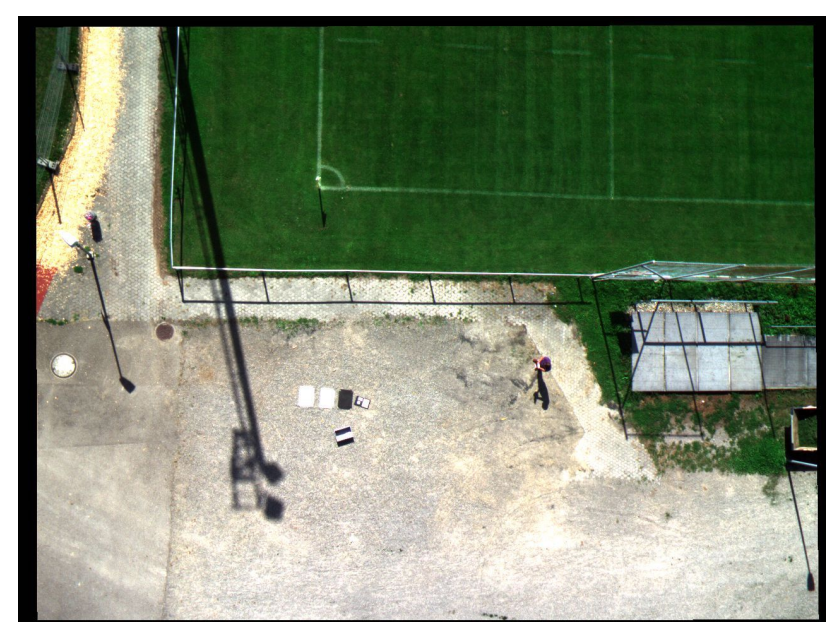

Figure 2. Micasense sample scene with reference panels (true color RGB, $37 \mathrm{~m}$ sensor altitude).
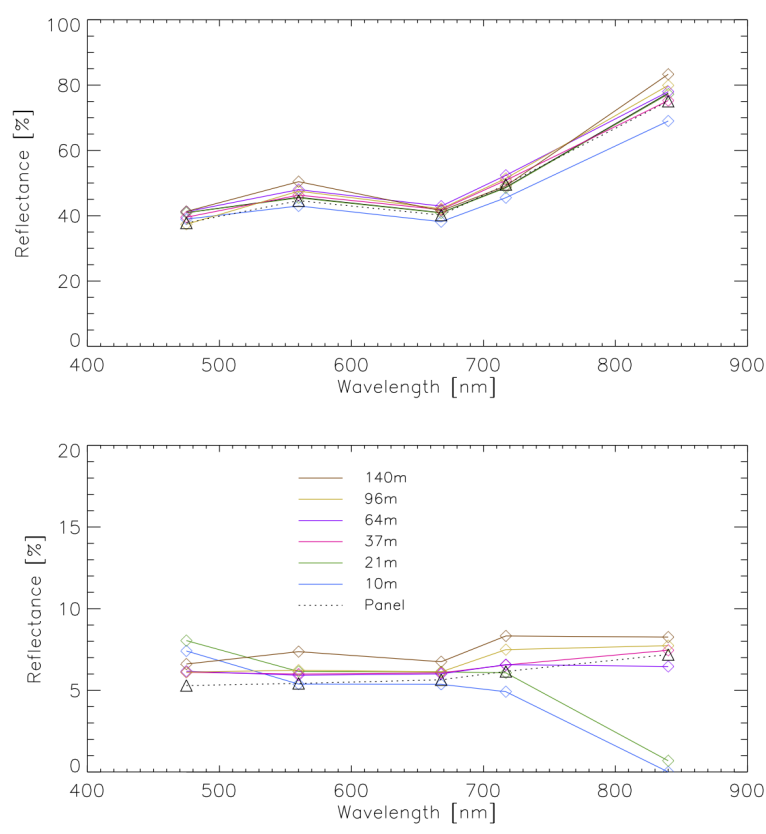

Figure 3. Micasense reflectance retrieval results using DROACOR atmospheric correction compared to raw image spectra. Top: bright target spectra, Bottom: dark panel spectra.

possible explanation may be related to the radiative transfer model. Increasing NIR radiance with flight altitude could be explained by the increasing total irradiance due to larger diffuse irradiance components, which may be occluded by nearby buildings or trees if flying below the horizon line.

Table 2. Averaged deviations between the dark reference panel reflectance and Micasense RedEdge reflectance outputs at various flight altitudes.

\begin{tabular}{c|c|c|c|c|c|c} 
Panel & $475 \mathrm{~nm}$ & $560 \mathrm{~nm}$ & $668 \mathrm{~nm}$ & $717 \mathrm{~nm}$ & $840 \mathrm{~nm}$ & Mean \\
\hline $5 \%$ & 2.23 & 2.72 & 1.73 & 1.91 & 4.08 & 2.54 \\
$50 \%$ & 1.45 & 0.77 & 0.52 & 0.94 & 2.72 & 1.28
\end{tabular}




\subsection{Headwall Nano Hyperspectral Instrument}

The second data set is a Headwall Nano data set acquired July 24th 2018 near Rapperswil, Switzerland (compare Figure 4). Data was taken at flight altitudes of $30 \mathrm{~m}, 100 \mathrm{~m}$, and $200 \mathrm{~m}$ above ground within a time span of 20 minutes. The solar zenith angle varied slightly between 27.4 and 27.8 degrees between acquisition times. A $28 \%$ and a $6 \%$ reference panel has been used for sensor inflight calibration using the data set $30 \mathrm{~m}$ above ground and a standard columnar water vapor amount of $2.5 \mathrm{~cm}$. The radiometric calibration constants have been retrieved from the $30 \mathrm{~m}$ flight altitude and are applied to the higher altitude data takes. The relative radiometric calibration of the Headwall system is assumed to be constant as the integration time is typically not changed during data acquisition.

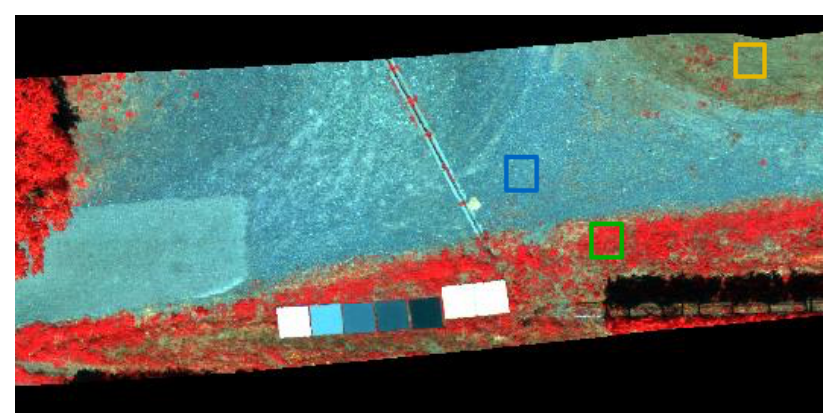

Figure 4. Hyperspec sample scene (30m flight altitude, RGB=NIR,Red,Green colors) with series of reference panels. The spectral samples positions are indicated by colored squares as of Figure 5.
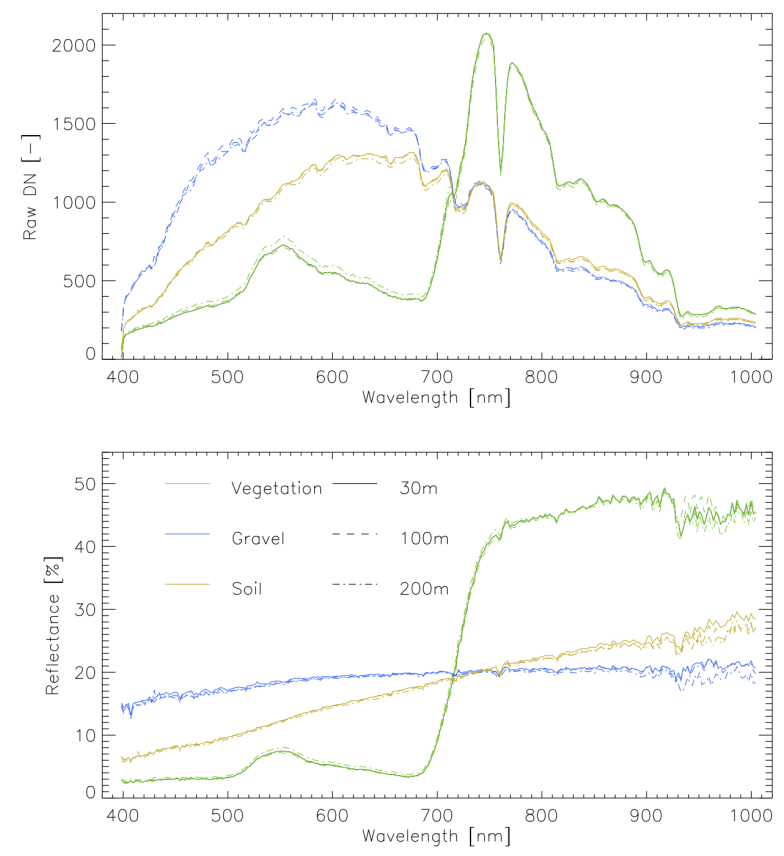

Figure 5. Hyperspec Nano sample spectra before and after atmospheric correction for three selected targets. The solid line is from $30 \mathrm{~m}$ above ground and the dashed lines are from $100 \mathrm{~m}$ and $200 \mathrm{~m}$ above ground, respectively.

Figure 5 shows typical resulting reflectance spectra radiative transfer based atmospheric correction at the locations indicated in Figure 4. The spectra have been averaged over $5 \times 5$ pixels for

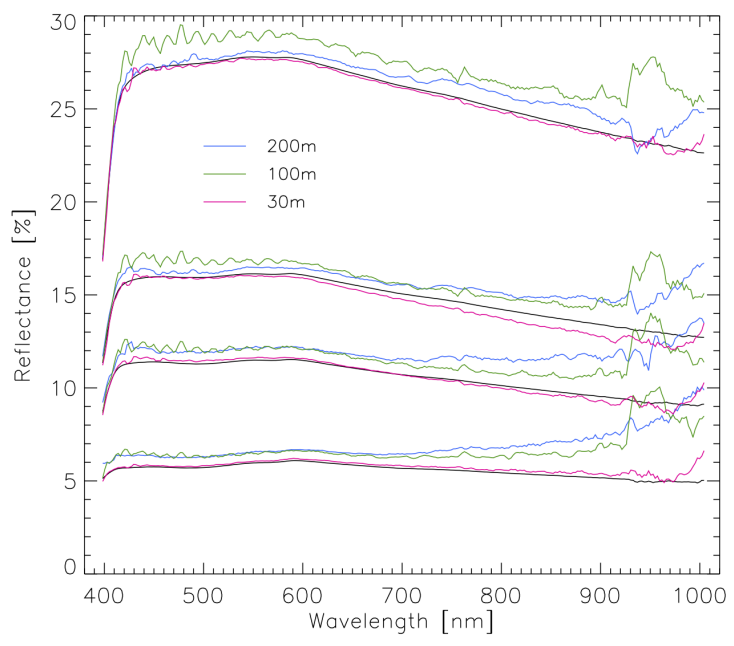

Figure 6. Hyperspec Nano panel spectra after empirical line correction using the $30 \mathrm{~m}$ data set for empirical line retrieval.

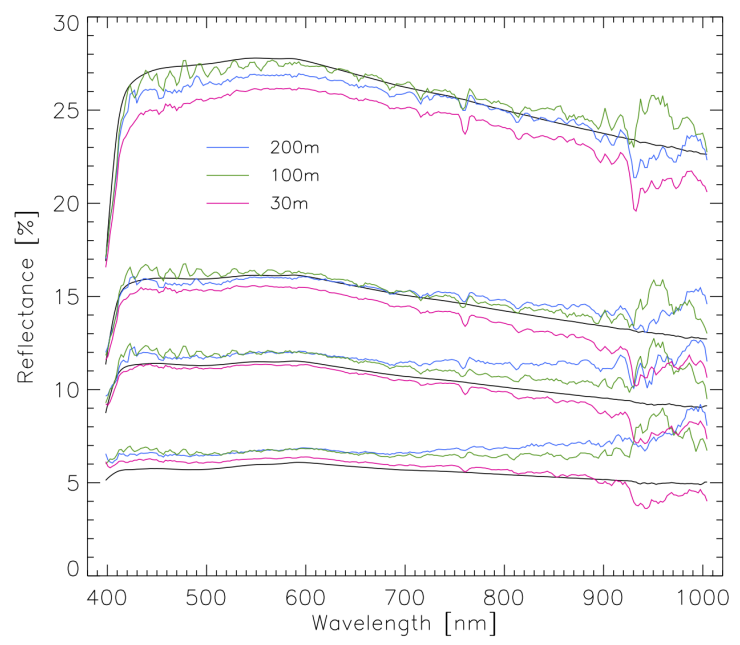

Figure 7. Hyperspec Nano panel spectra after Droacor correction using the $30 \mathrm{~m}$ data set for inflight radiometric calibration.

200m, 10x 10 pixels for $100 \mathrm{~m}$, and $33 \times 33$ pixels for the samples at $30 \mathrm{~m}$ above ground for validation. The averaging removes most of the spectral noise present in the data. Reflectances between flight altitudes agree within a small margin. Even within sensitive atmospheric absorption regions (710, 820, 940 $\mathrm{nm}$ ), the surface reflectance signatures are retrieved within a 5\% level of accuracy.

The same data set was corrected using the empirical line reflectance retrieval method (Farrand et al., 1994), with the same dark $6 \%$ reflecting panel in combination with the $28 \%$ panel (the brighter $50 \%$ panel could not be used due to saturation in portions of the visible range of the spectrum). Results for the empirical line correction are shown in Figure 6. The level of accuracy of this method was quantified by analyzing the differences of retrieved spectra of the $100 \mathrm{~m}$ and $200 \mathrm{~m}$ flight altitude data acquisitions to the reference panel. Significant differences are visible in the near infrared range of the spectrum, specifically for the dark object. This is consistent with the observations on the Micasense imagery. The water vapor bands can not be 
fully corrected based on the retrieved empirical relation. Furthermore, a systematic offset of the higher altitude data acquisitions of about $1-2 \%$ reflectance units can be observed.

The empirical line results are compared to the radiative transfer based correction as shown in Figure 7. The systematic differences for the $100 \mathrm{~m}$ and $200 \mathrm{~m}$ data acquisition are smaller for the DROACOR case, whereas the differences in the near infrared are comparable between the two methods. The RMS difference between panel spectra and retrieved reflectances is at $1.05 \%$ for the DROACOR outputs, whereas it is at $1.43 \%$ for the empirical correction.

\section{DISCUSSION}

The presented initial tests of the DROACOR method have shown the potential the model for physics based atmospheric correction of drone imagery. The comparison to the empirical reflectance retrieval methods are promising: in the investigated data sets, the radiative transfer based DROACOR performed on the same level of accuracy as empirical line correction or even lead to slightly better results. As the data sets were both acquired within a small time span (i.e., within 5 and 30 minutes, at noon time), these results are to be expected. For longer delays between panel based calibration and data aquisition, the radiative transfer based method theoretically has an advantage over any empirical method. However, this has still to be tested in practice by dedicated experiments or well suited multitemporal data sets.

The radiometric quality of drone based multispectral and hyperspectral sensors is constantly increasing and in some cases is approaching the quality of airborne systems. However, some sensors are affected by inconsistencies due to automatic sensor adjustments in flight or due to drifts in sensor calibration caused by temperature or pressure changes, as could be seen with the Micasense sensor in this analysis. In-depth knowledge about each supported sensor is required for an optimal reflectance retrieval. This fact becomes even more important if multitemporal data acquisitions are envisaged and the reflectance retrieval shall not be affected by temporal differences.

The observed differences in the NIR spectral range in the two presented data sets are puzzling and need some further investigation. The current correction procedure does not take adjacency effects into account. However, if considering the radiative path from sun to the adjacent ground to atmosphere and onto the observed pixel, considerable influence of adjacent reflectance properties are to be expected. Specifically dark objects having large reflectances in the NIR may show significantly brighter signatures than expected by the model. Therefore, the correction of the adjacency effects has to be revisited and the scattered diffuse irradiance may be modelled in a different way than currently done.

Another issue which has to be revisited is the retrieval of atmospheric parameters. The standard ATCOR method relies on the APDA method for water vapor retrieval (Schläpfer et al., 1998) and on the dark dense vegetation method (Kaufman, Tanré, 1996) for AOT retrieval. These methods are not applicable to airborne drone data as they both rely on the path from ground to sensor for parameter retrieval. For drone data, the signature of the irradiance is to be analyzed for the retrieval of both water vapor contents and aerosol optical thickness. Currently, only a rough retrieval method is implemented in DROACOR and new investigations into atmospheric parameter retrieval methods for the drone acquisition geometry are thus required.

Regarding topographic illumination corrections, one may think at first, that a standard illumination correction is not applicable to sensors at spatial resolutions in the centimeter-range, because topography is typically varying in the meter range only and smaller scale radiometric variations are strongly affected by target-specific shadowing effects. However, drone data is also often acquired at altitudes between 200 and $500 \mathrm{~m}$ what leads to a better coverage of the landscape but also leads to more obvious topography-induced illumination variations. Therefore, it is viable to add topographic corrections for drone processing as a last step of the processing. Such correction may also take into account the influence of cast shadow areas with methods as propose in (Schläpfer et al., 2018) and should include the BRDF characteristics of the surface for an accurate normalization.

Below cloud reflectance retrieval is a further issue to be addressed in the future. Data acquisitions on cloudy days are of interest for gap-less multitemporal monitoring and can often not be avoided, specifically in tropical regions. Currently, no methods are available on an operational basis which can solve below cloud situations on a physical basis. Even the use of in-field reference targets often is only of limited value as the illumination is highly variable with time. Thus, the whole processing needs to be re-thought. The use of on-board irradiance sensors may help in such situations, specifically if coupled with radiative transfer models. The development of improved solutions for such situations is a challenging broad field which needs further investigations.

\section{CONCLUSIONS}

A streamlined atmospheric correction procedure for drone based optical imagery has been designed and first tests have been presented. It has been shown in this contribution, that a radiative transfer based atmospheric correction can be successfully applied to both multispectral and hyperspectral drone imagery. UAV data acquisitions are often conducted over longer periods of the day. Then our physical approach will improve the analysis of the series of day-time images since ground brightness variations caused by varying illumination conditions can be compensated.

Further research has to be done with respect to the correct modelling of irradiance variations. A particularly difficult case is the treatment of shadow regions caused by trees or large objects, but also by cloud coverage. Also, the terrain correction is a topic which needs careful testing, specifically as no standard procedures for very high resolution imagery are defined yet in terms of irradiance correction.

The method shall be made publicly available on a commercial basis in the close future. The DROACOR software is being built specifically for operational processing of large volumes of drone data which includes fully automatic operation in a batch or cloud computing environment. The presented approach potentially will lead to higher consistency for large scale UAV data processing without the need of frequent deployment of reference panels in the field. The method will now be tested on further data sets including UAV based SWIR imaging spectrometers and additional modules such as terrain and irradiance correction will be added. 


\section{ACKNOWLEDGEMENTS}

AgriCircle AG, Rapperswil (Switzerland) ist thanked for providing the Headwall Nano data. The State College Wil is thanked for supporting the project and providing the Micasense RedEdge instrumentation.

\section{REFERENCES}

Aasen, H., Bolten, A., 2018. Multi-temporal high-resolution imaging spectroscopy with hyperspectral 2D imagers - From theory to application. Remote Sensing of Environment, 205, 374-389.

Aasen, H., Honkavaara, E., Lucieer, A., Zarco-Tejada, P., 2018. Quantitative Remote Sensing at Ultra-High Resolution with UAV Spectroscopy: A Review of Sensor Technology, Measurement Procedures, and Data Correction Workflows. Remote Sensing, 10(7), 1091-42.

Berk, A., Anderson, G. P., Acharya, P. K., Bernstein, L. S., Muratov, L., Lee, J., Fox, M., Adler-Golden, S. M., Chetwynd, J. H., Hoke, M. L., Lockwood, R. B., Gardner, J. A., Cooley, T. W., Borel, C. C., Lewis, P. E., 2005. MODTRAN 5: a reformulated atmospheric band model with auxiliary species and practical multiple scattering options: update. S. S. Shen, P. E. Lewis (eds), Algorithms and Technologies for Multispectral, Hyperspectral, and Ultraspectral Imagery X, SPIE Proc. 5425, 662-667.

Cattrall, C., Carder, K., Thome, K., Gordon, H., 2002. Solarreflectance based calibration of spectral radiometers. Geophysical Research Letters, 29(20), 1941-1944.

Eija Honkavaara, Ehsan Khoramshahi, 2018. Radiometric Correction of Close-Range Spectral Image Blocks Captured Using an Unmanned Aerial Vehicle with a Radiometric Block Adjustment. Remote Sensing, 10(2), 256-29.

Farrand, W. H., Singer, R. B., Merényi, E., 1994. Retrieval of apparent surface reflectance from AVIRIS data: a comparison of empirical line, radiative transfer, and spectral mixture methods. Remote Sensing of Environment, 47, 311-321.

Guo, Y., Senthilnath, J., Wu, W., Zhang, X., Zeng, Z., Huang, H., 2019. Radiometric Calibration for Multispectral Camera of Different Imaging Conditions Mounted on a UAV Platform. Sustainability, 11(4), 978-25.

Headwall Photonics Inc., 2019. Hyperspectral Imaging Sensors: Nano-Hyperspec. Headwall Nano-Hyperspec: https://www.headwallphotonics.com/hyperspectral-sensors (accessed Feb 2nd 2020).

Kaufman, Y. J., Tanré, D., 1996. Strategy for direct and indirect methods for correcting the aerosol effect on remote sensing: from AVHRR to EOS-MODIS. Remote Sensing of Environment, 55, 65-79.

Mayer, B., Kylling, A., Emde, C., Buras, R., Hamann, U., Gasteiber, J., Richter, B., 2019. libRadtran user's guide, Version 2.0.3. available from: http://www.libradtran.org/doc/libRadtran.pdf.

Micasense Inc., 2019. RedEdge-MX. Micasense RedEdge MX: https://www.micasense.com/rededge-mx (accessed Feb 2nd 2020).
Norsk Elektro Optikk AS, 2019. HySpex Turnkey Solutions. HySpex Mjolnir V-1240: https://www.hyspex.com (accessed Feb 2nd 2020).

Richter, R., Bachmann, M., Dorigo, W., Müller, A., 2006. Influence of the Adjacency Effect on Ground Reflectance Measurements. IEEE Geoscience and Remote Sensing Letters, 3(4), $565-569$.

Richter, R., Schläpfer, D., 2002. Geo-atmospheric processing of airborne imaging spectrometry data. Part 2: Atmospheric/Topographic Correction. International Journal of Remote Sensing, 23(13), 2631-2649.

Richter, R., Schläpfer, D., 2019. Atmospheric and Topographic Correction (ATCOR Theoretical Background Document). available from: https://www.reseapps.com/pdf/atcor_ATBD.pdf.

Richter, R., Schläpfer, D., Müller, A., 2011. Operational Atmospheric Correction for Imaging Spectrometers Accounting for the Smile Effect. IEEE Transactions on Geoscience and Remote Sensing, 49(5), 1772-1780.

Schläpfer, D., Borel, C., Keller, J., Itten, K., 1998. Atmospheric Pre-Corrected Differential Absorption Techniques to Retrieve Columnar Water Vapor. Remote Sensing of Environment, 65(3), 353-366.

Schläpfer, D., Hueni, A., Richter, R., 2018. Cast Shadow Detection to Quantify the Aerosol Optical Thickness for Atmospheric Correction of High Spatial Resolution Optical Imagery. Remote Sensing, 10(2), 25.

Schläpfer, D., Richter, R., 2011. Spectral Polishing of High Resolution Imaging Spectroscopy Data. 7th SIG-IS Workshop on Imaging Spectroscopy, Edinburgh, 1-7.

Schläpfer, D., Richter, R., Feingersh, T., 2015. Operational BRDF Effects Correction for Wide-Field-of-View Optical Scanners (BREFCOR). IEEE Transaction on Geoscience and Remote Sensing, 53(4), 1855-1864.

Sola, I., Gonzalez-Audicana, M., Alvarez-Mozos, J., 2016. Multi-criteria evaluation of topographic correction methods. Remote Sensing of Environment, 184, 247-262.

Specim Spectral Imaging Ltd., 2020. Specim FX Series. Specim AFX Series: https://www.specim.fi/afx (accessed Feb 2nd 2020).

Thompson, D. R., Guanter, L., Berk, A., Gao, B.-C., Richter, R., Schläpfer, D., Thome, K. J., 2018. Retrieval of Atmospheric Parameters and Surface Reflectance from Visible and Shortwave Infrared Imaging Spectroscopy Data. Surveys in Geophysics, 40(3), 333-360. 